\title{
STRANGULATED DIAPHRAGMATIC HERNIA IN THE NEONATAL PERIOD
}

\author{
BY \\ P. P. RICKHAM \\ From Alder Hey Children's Hospital, Liverpool
}

(RECEIVED FOR PUBLICATION APRIL 4, 1955)

Strangulation of a diaphragmatic hernia was first described by Paré in 1575 . It is a rare complication of this type of hernia; Hoffman, Levy, Sole, and Lewitan (1954) could only find 76 cases in the literature and added a case of their own. Most of the earlier instances of strangulation occurred in herniae of traumatic origin, but in recent years there has been a higher proportion in congenital herniae.

The mortality rate for strangulated diaphragmatic herniae has always been very high, $88.8 \%$ before 1920 and $43.7 \%$ in later cases (Carter and Giuseffi, 1948). The mortality rate is still high because of the vagueness of symptoms, difficulties of diagnosis, and the resulting delay in operation.

The youngest case previously reported was a girl, 6 weeks old, who had vomited for a week and was shocked and cyanosed on admission. The sigmoid colon had herniated in the left pleural cavity. On reduction it was found that a length of 3 in. was strangulated but viable. The diaphragmatic defect was repaired (Cooke and Corner, 1949). No case of strangulation of a diaphragmatic hernia during the neonatal period has previously been reported, though diaphragmatic herniae, usually presenting with severe respiratory embarrassment during the first few days of life, are not uncommon. We have operated upon eight such cases during the last four years. That strangulation does not occur more often may be explained, in part, by the fact that most of the infants die as a result of respiratory embarrassment in the first few days of life unless operated upon, and there is therefore little time for strangulation to occur. Another cause for the rarity of this complication may be the relatively large size of the pleuro-peritoneal canal in these babies.

This case is of interest because it is the first recorded of a strangulated diaphragmatic hernia in a newborn infant and because the diagnosis was made pre-operatively.

\section{CASE History}

Anthony S. was admitted to the Neonatal Surgical ${ }^{\circ}$ Unit at Alder Hey Children's Hospital on April $9, \vec{c}$ 1954, when 2 weeks old.

The mother's pregnancy and labour had been normal. The child's birth weight was $6 \mathrm{lb} .12 \mathrm{oz}$. and he had $\vec{\bullet}$ been well until six hours before admission, when hec had had a convulsion and had become cyanosed. $\mathrm{He}^{\text {. }}$ had vomited copiously several times and the vomitus. had been bile-stained.

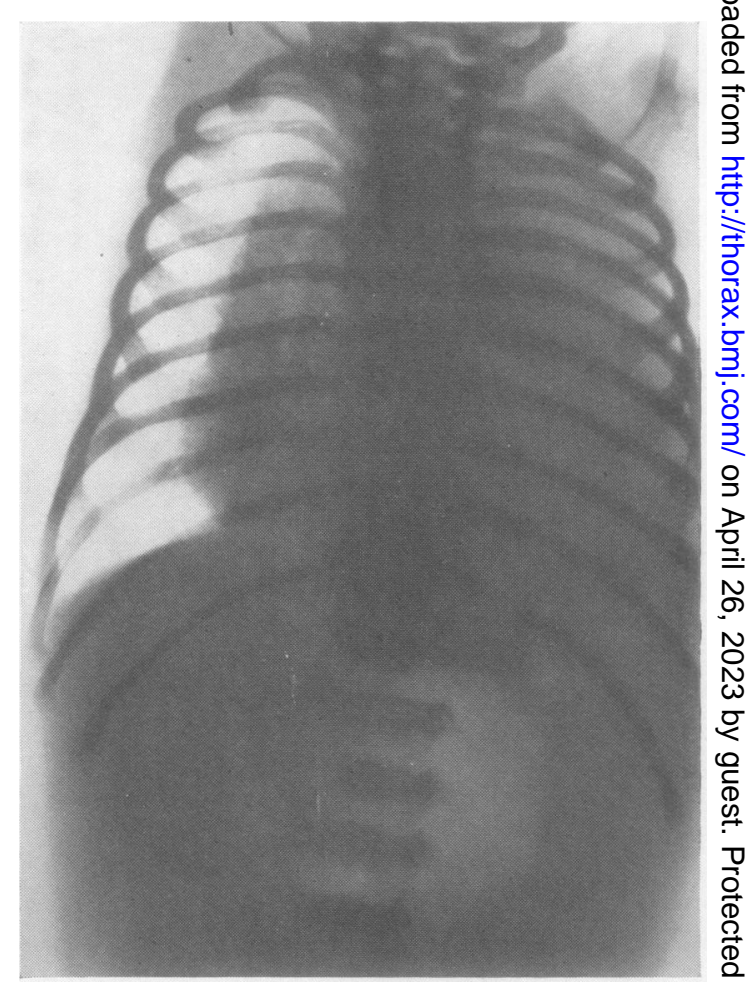

FIG. 1.-Radiograph of the chest and abdomen showing uniform opacity of the left chest, displacement of the mediastinum, and no abdominal gas apart from the stomach bubble. 
On admission he was very shocked and cyanosed; respiration was rapid and shallow. Respiratory movements were confined to the right side of the chest; there was complete dullness to percussion and absence of breath sounds over the left chest, and it was noticed that the mediastinum had shifted towards the right. The abdomen was scaphoid and uniformly dull to percussion. The rectum was found to be empty of faeces, and when the examining finger was withdrawn a considerable amount of blood and mucus was passed.

Radiography of the chest and abdomen showed uniform opacity of the left chest with displacement of the mediastinum towards the right side. With the exception of a small bubble in the stomach, there was no gas in the abdomen (Fig. 1)

An intravenous infusion was started, a catheter was passed into the stomach, and a small quantity of bilestained fluid was aspirated by continuous low-pressure suction.

Ten hours after the onset of the symptoms the child was anaesthetized using endotracheal nitrous oxide, oxygen, and minimal ether anaesthesia with intravenous succinylcholine injections and controlled respiration (Dr. J. G. Rees).

A fine needle was inserted into the left chest through the eighth intercostal space in the mid-axillary line and $5 \mathrm{ml}$. of milky fluid was aspirated. The abdomen was opened through an oblique incision from the point where the anterior axillary line crosses the left costal margin to a point in the midline of the abdomen just above the umbilicus. The peritoneal cavity was opened and a large quantity of milky fluid, similar to that aspirated from the left chest, was sucked out. The abdomen contained only the liver, spleen, stomach, duodenum, and sigmoid colon; all the rest of the small and large intestine had herniated through a patent left pleuro-peritoneal canal into the chest. The herniated intestines were pulled down into the abdomen with some difficulty, as the gut was distended with fluid and tightly wedged through a narrow, constricting hiatus. The gut was blackish-blue, but the surface was glistening. It was wrapped in warm saline packs and quickly regained its normal colour. There was a large amount of milky fluid in the right pleural cavity which was removed by suction. The left lung was collapsed and was expanded with some difficulty by the anaesthetist. A small, selfretaining catheter was inserted into the left pleural cavity through the ninth intercostal space in the posterior axillary line for underwater drainage. The pleuroperitoneal hiatus was repaired with two layers of interrupted silk sutures without difficulty.

The child stood the operation well.' During the operation $75 \mathrm{ml}$. of blood was given by transfusion. Post-operatively, intravenous infusions of $5 \%$ glucose solution, $5 \mathrm{ml}$. per hour, were given for 24 hours. Lowpressure gastric suction was continued. The infant was nursed in a surgical incubator in an atmosphere of $70 \%$ oxygen concentration and $100 \%$ humidity at $85^{\circ} \mathrm{F}$. A radiograph taken six hours after operation showed that the left lung had completely expanded (Fig. 2). Graduated oral feeding was started the day following

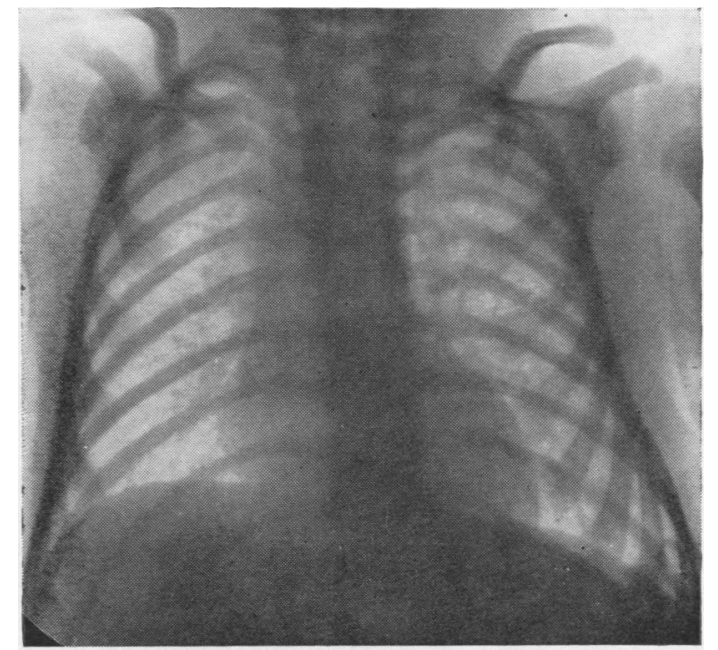

FIG. 2.-Radiograph of the chest six hours after operation, showing complete expansion of left lung.

operation and the child was discharged home two weeks after admission. He has since been followed up and remains well.

\section{Discussion}

Only a few points need some elaboration. The diagnosis was made by a process of exclusion. The infant vomited bile and was therefore likely to be suffering from intestinal obstruction below the ampulla of Vater. He was very shocked and had passed blood and mucus by rectum. It was therefore thought that he suffered from intestinal strangulation. There was a large opacity on the left side of the chest which caused mediastinal displacement and respiratory embarrassment. Intraabdominal strangulation coupled with pleural effusion, perhaps due to pneumonia, had to be considered, but the short history, the scaphoid abdomen, and the almost complete absence of intestinal gas on the radiograph was against this double diagnosis. The absence of any intestinal shadows in the chest radiograph, considered to be characteristic of strangulated diaphragmatic hernia (Pearson, 1953), was somewhat puzzling. It was, however, considered that if there was interference with the venous return only the intestine would rapidly fill with fluid and the pleural and abdominal cavity with transudate. Thirty per cent. of all cases of strangulated diaphragmatic herniae have free fluid in the chest (Carter and Giuseffi, 1948); in most of the reported cases the fluid has been blood-stained.

We had hoped that aspiration of the chest would confirm the diagnosis. The aspirated fluid, however, was not blood-stained, but it was unlike pleural 
exudate and resembled chyle; it was therefore thought to be caused by obstruction of the intestinal lymphatics. $\mathrm{W}$ hen the strangulated intestines were closely inspected at operation, it was seen that the lymphatic vessels stood out as milky-white threads against the blackish-blue background of the strangulated gut and were oozing chyle.

Formerly we approached diaphragmatic herniae in infants through the chest; during the last four years we have returned to the abdominal route popularized by Ladd and Gross (1940), using the oblique incision described above. This incision gives an excellent exposure of the pleuro-peritoneal canal, and, as it runs in the line of the nerves to the musculature, does not cause any weakness of the abdominal wall. Difficulty in closing the peritoneum after the intestines have been returned from the chest to the abdomen has not bee殿 encountered with the complete muscular relaxation which our anaesthetists are able to obtain. Af intercostal underwater drain has, in our experienceep facilitated expansion of the lung. The rapidity with which such a lung, which has never beep used, can fully expand is shown by the secones radiograph.

I should like to thank Miss Isabella Forshall for her, help in preparing this paper, and Dr. J. Sytner foo referring the case.

\section{REFERENCES} Carter, B. N., and Giuseffi, J. (1948). Ann. Surg., 128, 210. Hoffman, W., Levy, M. L., Sole, E., and Lewitan, A. (1954). Surg, Chicago, 69, 125

Ladd W.' New Engl. J. Med., 223, 917 Ladd, W. E., and Gross, Reuvres, lib. 10, cap. 30, Par is: G. Buon. Pearson. S. (1953). Arch. Surg., Chicago, 66, 155. 